\title{
Panorama dos Bacharelados Interdisciplinares no Brasil
}

\section{Overview of Interdisciplinary Bachelor's Degrees in Brazil}

\author{
Francisco Nilton Gomes de Oliveira' \\ Leandro Silveira Ferreira ${ }^{2}$ \\ Lidiane Bittencourt Barroso ${ }^{3}$
}

\section{RESUMO}

Este artigo situa e contextualiza os cursos de Bacharelado Interdisciplinar (BI), no Brasil, e objetiva sinalizar para gestores de ensino superior, pesquisadores e demais interessados no tema o cenário em 2017 da oferta desses cursos. Tais programas de bacharelado interdisciplinar tiveram início com a implantação da Reestruturação e Expansão das Universidades Federais (REUNI), em 2007, e foram implantados com o objetivo de confrontar o modelo enciclopédico, redimensionando as novas formações universitárias em uma perspectiva de transversalização nas áreas de conhecimento das universidades brasileiras. Metodologicamente, a pesquisa baseou-se em documentos basilares sobre o tema em discussão e na plataforma e-MEC - sistema eletrônico de monitoramento dos processos que regulamentam a educação superior no Brasil. Os resultados foram obtidos em pesquisa documental e dados quantificados e categorizados para a compreensão do panorama no Brasil. Conclui-se que apesar dos grandes esforços para mudar a formação do alunado brasileiro, o BI ainda é uma realidade incipiente no país. As universidades públicas concentram o maior número de $\mathrm{BI}$, havendo resistência geral à implantação de novos modelos de formação no ensino superior. Houve avanços dos cursos BI, reconhecendo as metodogias inovadoras nesta formação pegagógica dentro de uma abordagem interdisciplinar mais plural, mesmo assim ainda há desafios e resistências, entre docentes e estudantes, implicadas a partir de paradigmas conservadores do modelo tradicional de formação.

Palavras-chave: Educação Superior. Instituição de Ensino Superior. Interdisciplinariedade. Plataforma eletrônica e-MEC.

\footnotetext{
1 Universidade Federal de Santa Maria | niltonoliveira@superig.com.br

2 Universidade Federal de Santa Maria - Colégio Técnico Industrial | leandro.ferreira@ctism.ufsm.br

3 Universidade Federal de Santa Maria - Colégio Técnico Industrial | lidianebarroso@gmail.com
} 


\title{
Panorama dos Bacharelados \\ Interdisciplinares no Brasil
}

Overview of Interdisciplinary Bachelor's Degrees in Brazil

\begin{abstract}
This article situates and contextualizes the Interdisciplinary Bachelor (BI) courses in Brazil, and aims to signal to higher education managers, researchers and others interested in the theme the scenario in 2017 of the offer of these courses. Such interdisciplinary baccalaureate programs started with the implementation of the Restructuring and Expansion of Federal Universities (REUNI), in 2007. BI courses were implemented in Brazil with the objective of confronting the encyclopedic model, resizing the new university backgrounds in a perspective of transversalization in the areas of knowledge of Brazilian universities. Methodologically, the research was based on basic documents on the topic under discussion and on the e-MEC platform - electronic system for monitoring the processes that regulate higher education in Brazil. The results were obtained from documentary research and quantified and categorized data for understanding the panorama in Brazil. It is concluded that despite the great efforts to change the formation of Brazilian students, $\mathrm{BI}$ is still an incipient reality in the country. Public universities concentrate the largest number of $\mathrm{BI}$, with general resistance to the implementation of new training models in higher education. There have been advances in BI courses, recognizing the innovative methodologies in this pedagogical training within a more plural interdisciplinary approach, yet there are still challenges and resistance, between teachers and students, implied from conservative paradigms of the traditional training model.
\end{abstract}

Keywords: College Education. Higher Education Institution. E-MEC electronic platform. 


\section{Introdução}

O presente trabalho tem como objetivo realizar um panorama nacional sobre a situação, em 2017 , dos cursos de Bacharelados Interdisciplinares (BI), bem como resgatar a história da implantação e implementação dos cursos no país. Trata-se de uma investigação sobre a educação superior com o foco na busca por dados para contribuir com o Ministério da Educação (MEC), Coordenação de Aperfeiçoamento de Pessoal de Nível Superior (CAPES), pesquisadores e demais interessados. $O$ estudo dessa temática nasce de uma inquietude dos autores, que vislumbram compreender o cenário, em 2017, dos Bacharelados Interdisciplinares no Brasil.

Historicamente, salienta-se que a proposta dos cursos de BI é algo recente na educação no país, pois altera o quadro de disciplinas que compõem as matrizes curriculares dos cursos superiores. Portanto, apresenta grandes desafios para a sua consolidação, devido à dificulade de ruptura do modelo tradicional das universidades brasileiras, especialmente, suas arquiteturas acadêmicas postas no Brasil desde o final do século XIX e início do século XX. Próximo desse período (metade do séc. XIX), ocorreu uma imposição da ciência conhecida atualmente. Nessa era ganha destaque o método científico como o caminho para se produzir conhecimento, fundamentada no positivismo e no experimento (KUHN, 2003).

De acordo com a história, o início dos cursos superiores no Brasil deu-se no ensino profissionalizante e era direcionado para a elite, cujos membros faziam parte da Corte portuguesa e tinham estabelecido residência no país. Para Favero (2006), o processo de colonização foi determinante para que a elite ainda enviasse seus familiares à formação de nível superior na Europa.

No início do século XX, a Universidade ganha forma pela integração de antigas faculdades profissionalizantes oriundos da Era Colonial, especialmente, no período Joanino (PAIM, 1981). No entanto, outra mudança transfigura-se em meados dos anos 1940 a 1960, em que há uma preocupação das universidades com uma formação mais acadêmica e científica no Brasil.

A partir da década de 1960, a ideia de interdisciplinaridade foi apresentada como uma precursora da criticidade. A partir da busca constante por respostas até os limites do conhecimento simplificador, dicotômico e disciplinar da ciência moderna e clássica rompeu-se o paradigma do modelo tradicional na formação do estudante na educação superior. Isso tinha como princípio a negação do pressuposto básico do conhecimento objetivo de que havia um vazio nas fronteiras de cada disciplina (ALVARENGA et al. , 2011).

A partir dessa acepção, a discussão da interdisciplinaridade emergiu no pais, provocando novos saberes na formação pedagógica, na arquitetura acadêmica e na concepção dos modelos formativos nas Universidades. Em meados do ano 1960, houve uma mudança paradigmática no que diz respeito à lógica na formação da educação superior. Nesse momento, as fronteiras de cada disciplina são refutadas cientificamente e cria-se uma discussão nos limítrofes, associando novos conceitos que vão ao encontro da interdisciplinaridade.

A Interdisciplinaridade tem como finalidade responder aos problemas criados pelo próprio avanço da ciência moderna, quando esta última tem como característica ser fragmentadora e simplificadora da realidade. Segundo Santos:

[...] a simplificação das leis constitui uma simplificação arbitrária da realidade que confina a um horizonte mínimo; a noção de lei tem vindo a ser parcial e sucessivamente substituída pelas noções de sistema, de estrutura, de modelo e, por último, pela noção de processo. Assim, o declínio da hegemonia da legalidade é concomitante do declínio da hegemonia da causalidade (SANTOS, 2008, p. 52).

Para Heckhausen (1972), para especificar o sentido vago relacionado ao termo interdisciplinaridade, tem-se que saber antes o que é uma disciplina, a partir da apresentação de critérios para a sua 
definição: tais como a existência de um objeto próprio; de um campo de conhecimento definido; de um nível de integração teórica; de métodos próprios de investigação; de instrumentos epistemológicos de análise; aplicação prática; e, contingências históricas que a dinamizam. Nessa perspectiva, o autor distinguiu seis tipos de relações interdisciplinares, em ordem ascendente de maturidade:

Interdisciplinaridade heterogênea: esforços de caráter enciclopédico - busca combinar, notadamente no ensino, programas disciplinares diferenciados tendo em vista contrabalançar os efeitos da especialização.

Interdisciplinaridade composta: aptidão técnica em tomar problemas complexos colocados pela sociedade como objeto comum a várias disciplinas (fome, a degradação das paisagens, o caos urbano).

Pseudointerdisciplinaridade: falsa concepção de existência de uma interdisciplinaridade intrínseca.

Interdisciplinaridade auxiliar: trocas ou empréstimos nos métodos e nas técnicas de pesquisa entre disciplinas.

Interdisciplinaridade complementar: regiões fronteiriças de certas disciplinas pertencentes aos mesmos campos que se imbricam parcialmente.

Interdisciplinaridade unificadora: coerência cada vez mais estreita dos campos de estudo de duas disciplinas (HECKHAUSEN, 1972, p.32).

De acordo com essa acepção, a nossa formação, necessária ao enfrentamento dos desafios, necessita transcender a especialidade em torno de uma única área do saber e do fazer humanos. 0 intenso avanço científico e tecnológico alcançado na atualidade modifica profundamente os horizontes em que nos inserimos. Logo, a realidade, em torno da qual nos movimentamos, apresenta-se cada vez mais multifacetada, híbrida, exigindo múltiplos olhares investigativos em sua análise, interpretação e compreensão. Sob todas estas perspectivas, faz-se urgente refletir a interdisciplinaridade como postura científica e epistemológica, e suas condições de possibilidade de ampliação dos horizontes cognitivos e interpretativos do ser humano diante dos desafios sociais, políticos, econômicos, culturais, ambientais, científicos e tecnológicos (ALVARENGA et al., 2011).

Parafraseando Alvarenga et al. (2011), ao considerar que o mundo está em constante mudança, não há garantias de empregabilidade estável e segura como em outros tempos. Neste cenário, o que a Universidade pode fazer, é conferir consistência à formação humana e técnica do acadêmico, possibilitando-Ihe uma sólida capacidade de reflexão e análise do contexto de mundo em que está inserido, e a implicância disso sobre a totalidade de sua existência. Paralelamente às demandas pela especialização, o mercado requer das universidades uma oferta de conhecimento intelectual que, é cada vez mais reconhecida pelos analistas do mercado.

A experiência de cursos interdisciplinares no Brasil sinaliza uma mudança para uma prática pedagógica que permita o alunado tecer costuras em diferentes áreas de conhecimento. Isso possibilitará uma formação diferenciada no que diz respeito para uma formação mais generalista e apta ao mercado de trabalho, do qualemerge um profissional com múltiplos conhecimentos.

Os cursos de Bl estão definidos no documento "Referenciais Orientadores para os Bacharelados Interdisciplinares e Similares” (BRASIL, 2010) como programa de formação em nível de graduação - que levam à diplomação, com organização disposta em grandes áreas do conhecimento. Entendem-se como grande área do conhecimento: as práticas, os saberes, as tecnologias e os campos do conhecimento.

Este documento (BRASIL, 2010) mostra que, no país, a concepção da formação em ciclos é proveniente de um período no qual houve um aumento gradativo de matrículas na educação superior, especialmente, no setor privado nos anos 1990. De acordo com essa concepção, no ano de 2007, o governo federal implantou um programa de apoio ao REUNI e, nesse mesmo período, emergiu o 
movimento de interiorização da implantação e implementação dos Institutos Federais de Educação Superior (IFES). O REUNI estabeleceu uma nova jornada nas Instituições de Ensino Superior (IES), fazendo com que as mesmas pudessem abrir espaço para uma nova estruturação, cujas dimensões atingem a ampliação da oferta da educação superior pública, a reestruturação acadêmica curricular, seu compromisso social e mobilidade interinstitucional.

Os cursos de BI são elucubrados, em 2006, pela Universidade Federal da Bahia (UFBA) através de docentes vinculados à educação superior, em uma iniciativa de incrementar uma proposta pedagógico-metodológica inovadora, como uma forma de romper com o paradigma enciclopédico impetrado nas universidades brasileiras desde o século XIX. Eles emergem a partir da implantação do REUNI, não somente na perspectiva da reestruturação e expansão do ensino superior, mas também com a intenção de inovar nas estruturas acadêmico-pedagógicas das IES no Brasil. Eles surgem com a intenção de suprir necessidades educacionais e oportunizar aos indivíduos que não tiveram acesso ao ensino superior, devido às condições de vida sejam estas: questões socioeconômicas, geográfico-territoriais e ambientais.

No condão do REUNI, outras IES implantaram cursos de BI em diferentes áreas do conhecimento, tais como: Fundação Universidade Federal do ABC (UFABC), Universidade Federal de Juiz de Fora (UFJF), Universidade Federal do Sul da Bahia (UFSB), dentre outras. Salienta-se que as IES foram implantadas e implementadas com uma arquitetura pedagógica e metodológica diferenciada, repensando as opções curriculares. Assim, partindo de matrizes curriculares mais dinâmicas, os cursos de BI confrontaram o modelo enciclopédico de outros cursos superiores e redimensionaram as novas formações universitárias numa perspectiva de transversalização nas áreas do conhecimento nas universidades brasileiras.

A arquitetura acadêmica vigente no Brasil incorpora uma profusão tecnológica (bacharelado, licenciatura, habilitação, ênfase, tecnólogo, etc.), evidenciando-se um modelo já consolidado ao longo dos anos, com currículos estreitos e fechados, sem atender uma formação mais generalista do alunado. Os cursos BI vêm com a intenção de suprir essas necessidades que são exigidas no mundo contemporâneo.

Segundo Almeida Filho (2014), a interdisciplinaridade conduz a três sentidos ou modalidades: Interface entre campos disciplinares, enriquecendo objetos específicos de conhecimento (p. ex. Antropologia Social; Sociologia Jurídica);

Fusão de disciplinas, resultando em objetos também fusionados (p.ex. Físico-Química ou Bioinformática);

Uso de múltiplas abordagens, oriundas de distintos campos disciplinares, para produzir conhecimento ou ação sobre um problema concreto (e complexo).

Nesse último caso, abrem-se perspectivas ou pertinências de passagem ou trânsito entre distintos campos disciplinares e interdisciplinares, no processo complexo de formação de sujeitos, que têm sido designadas como Transdisciplinaridade (ALMEIDA FILHO, 2014, p.22).

Enquanto isso, a ruptura paradigmática do modelo enciclopédico só ocorreu a partir da implementação de novas estratégias pedagógicas nos cursos de licenciaturas, bacharelados e tecnológicos. Caso não ocorrea essa prática a formação do alunado é prejudicada, porque está centrada no docente. Os cursos de BI são uma oportunidade das IES redefinirem modelos de estratégias pedagógicas, bem como aproximarem a inovação na formação acadêmica, como é exigido na "Declaração Mundial sobre a Educação Superior no Século XXI: Visão e Ação" (UNESCO, 1998).

Os cursos de BI preparam-se para ser uma mola propulsora da ruptura do paradigma do modelo enciclopédico, propiciando ao alunado uma formação mais generalista e interdisciplinar, e aproximando-o da realidade do mercado de trabalho do século XXI. 


\section{Metodologia}

O estudo é oriundo de uma pesquisa documental-descritiva. Parafraseando Moreira e Caleffe (2006), compreendem-se por uma pesquisa documental-descritiva aquela que se baseia em documentos, podendo ser elaborada e descrita por meio da dissertação dos fatos achados. O panorama foi baseado em coleta de informações e de documentos legislativos do MEC, tais como: atos regulatórios, portarias de reconhecimento e autorização, procedentes de um banco de dados de domínio público, fornecido pela plataforma eletrônica e-MEC.

O e-MEC é um sistema eletrônico desenvolvido e disponibilizado ao usuário de forma gratuita, o qual gerencia informações relacionadas à regulação, processos de avaliação e à supervisão de instituições que fazem parte do sistema federal de ensino. Além disso, o cadastro e-MEC disponibiliza indicadores de qualidade dos cursos, banco de avaliadores (Basis) e resultados do Exame Nacional de Desempenho de Estudantes (ENADE) (BRASIL, 2007).

Através de verificação realizada na plataforma eletrônica e-MEC de instituições e cursos de educação superior, obteve-se dados dos indicadores que fornecem informações sobre os mais variados cursos de nível superior. Além disso, a plataforma é uma fonte oficial e única, com dados que estão de acordo com os atos autorizativos das faculdades, centros universitários e universidades brasileiras (BRASIL, 2007).

Os dados do cadastro dos cursos de BI foram coletados através da consulta avançada na plataforma eletrônica e-MEC. Os procedimentos utilizados no estudo restringiram a busca no banco de dados, ao se nominar, por cursos de graduação, que continha a pesquisa exata: interdisciplinar.

Como resultado a própria plataforma eletrônica e-MEC elencou todos os dados instados para o estudo, uma tabulação subdividida em categorias (IES, sigla, nome do curso, grau, modalidade, índices, vagas autorizadas, data início funcionamento, situação). A modalidade de ensino também pode ser restringida, optando-se por presencial ou a distância. Além disso, pode-se pesquisar o grau e a situação dos cursos e, até mesmo, o local de funcionamento e a sua gratuidade.

Nessa pesquisa, não houve restrição quanto a modalidade e à situação, no entanto, no campo para informar o grau, marcou-se a opção bacharelado, já que o escopo do estudo é o panorama dos cursos de bacharelados interdisciplinares. Cabe relatar que também existem cursos de licenciatura interdisciplinares, os quais não foram listados aqui.

\section{Resultados e discussão}

Neste tópico mostram-se os resultados obtidos a partir da pesquisa documental na plataforma eletrônica e-MEC e seus dados quantitativos de frequência. Em resumo, a determinação de quantos cursos de $\mathrm{Bl}$, cuja tipologia já foi mencionada, presentes nas instituições de ensino superior. Com base nessa tabulação dos dados, oriunda da consulta ao cadastro dos cursos de $\mathrm{Bl}$, e da reflexão dos autores, analisou-se o panorama nacional, fundamentando-o em documentos de domínio público, como os referenciais orientadores e as portarias. Salienta-se que as informações estão sempre sendo atualizadas na plataforma eletrônica e-MEC (BRASIL, 2017a).

As categorias analisadas foram: número de instituições de ensino superior que ofertaram os cursos de $\mathrm{Bl}$; os respectivos atos regulatórios (criação do curso presencial, autorização e reconhecimento); a espacialização regional versus o número de vagas autorizadas; o conceito de curso até o ano de 2017. Estas informações foram categorizadas, de acordo com a consulta avançada, por cursos de bacharelado que continham, no seu cadastro, o nome exato interdisciplinar.

Ao acessar a plataforma eletrônica e-MEC foram obtidos um total de 66 cursos de Bacharelados Interdisciplinares, com predominância de oferta em instituições públicas e somente um curso interdis- 
ciplinar em Ciências do Trabalho ofertado por uma instituição privada: a Escola DIEESE de Ciências do Trabalho. A maioria dos cursos encontrados, isto é, 71,2\% são procedentes das 13 instituições federais que participaram do grupo de trabalho ou do envio de sugestões para a elaboração dos referenciais orientadores para cursos de BI e similares (BRASIL, 2010), somando um subtotal de 47 cursos. Completam a listagem outras 5 instituições federais que respondem por 27,3\%, ou seja, 18 cursos de BI. Com isso, elaborou-se a tabela 1, na qual se apresenta um resumo dos dados quantitativos obtidos a partir da consulta a plataforma eletrônica e-MEC (BRASIL, 2017a).

As instituições como a Universidade Federal do Rio de Janeiro (UFRJ), a Universidade da Integração Internacional da Lusofonia Afro-brasileira (UNILAB) e a Universidade Federal da Fronteira Sul (UFFS) participaram do grupo de trabalho para a elaboração dos Referenciais Orientadores, entretanto não apresentaram cursos de BI durante a realização desta pesquisa.

\section{Quadro 1 - Quantidade de cursos bacharelados interdisciplinares (BI) por IES}

\begin{tabular}{|c|c|c|}
\hline Instituição de Ensino Superior (IES) & Sigla & $\begin{array}{c}\text { Quantidade de } \\
\text { cursos de BI }\end{array}$ \\
\hline Escola DIEESE de Ciências do Trabalho & DIEESE & 1 \\
\hline \multicolumn{2}{|l|}{1 Instituição Privada que oferta curso interdisciplinar. } & $1(1,5 \%)$ \\
\hline Universidade Federal de Santa Catarina & UFSC & 1 \\
\hline Fundação Universidade Federal do ABC & UFABC & 3 \\
\hline Universidade Federal de São João del Rei & UFSJ & 2 \\
\hline Universidade Federal de Juiz de Fora & UFJF & 2 \\
\hline Universidade Federal da Bahia & UFBA & 8 \\
\hline Universidade Federal do Rio Grande do Norte & UFRN & 1 \\
\hline Universidade Federal do Oeste do Pará & UFOPA & 9 \\
\hline Fundação Universidade Federal do Pampa & UNIPAMPA & 1 \\
\hline Universidade Federal de Alfenas & UNIFAL-MG & 2 \\
\hline Universidade Federal Rural do Semi-Árido & UFERSA & 7 \\
\hline Universidade Federal dos Vales do Jequitinhonha e Mucuri & UFVJM & 4 \\
\hline Universidade Federal do Recôncavo da Bahia & UFRB & 3 \\
\hline Universidade Federal do Maranhão & UFMA & 4 \\
\hline \multicolumn{2}{|l|}{13 Instituições Federais que participaram do grupo de trabalho. } & $47(71,2 \%)$ \\
\hline Universidade Federal do Pará & UFPA & 1 \\
\hline Universidade Federal do Rio Grande do Sul & UFRGS & 1 \\
\hline Universidade Federal de São Paulo & UNIFESP & 2 \\
\hline Universidade Federal do Oeste da Bahia & UFOB & 2 \\
\hline Universidade Federal do Sul da Bahia & UFSB & 12 \\
\hline \multicolumn{2}{|l|}{5 Instituições Federais que também aderiram a nova arquitetura. } & $18(27,3 \%)$ \\
\hline Total de cursos de BI ofertados nas 19 IES & & $66(100 \%)$ \\
\hline
\end{tabular}

Fonte: Elaborada pelos autores, consulta plataforma eletrônica e-MEC (BRASIL, 2017a)

A seguir estão descritas as respectivas denominações de cada curso de BI dessas IES, listadas quantitativamente no quadro 1: a Universidade Federal de Santa Catarina (UFSC) com 1 curso interdisciplinar em Mobilidade; a Fundação Universidade Federal do ABC (UFABC) com 3 cursos, 2 em Ciência e Tecnologia e 1 em Ciências e Humanidades; a Universidade Federal de São João del 
Rei (UFSJ) com 2 cursos, 1 em Ciência e Tecnologia e 1 em Biossistemas; a Universidade Federal de Juiz de Fora (UFJF) com 2 cursos, 1 em Artes e Design e 1 em Ciências Humanas; a Universidade Federal da Bahia (UFBA) com 8 cursos, 2 em Artes, 2 em Ciência e Tecnologia, 2 em Humanidades e 2 em Saúde; a Universidade Federal do Rio Grande do Norte (UFRN) com 1 curso em Ciência e Tecnologia; a Universidade Federal do Oeste do Pará (UFOPA) com 9 cursos diferentes, 1 em Ciência e Tecnologia, 1 em Ciência e Tecnologia das Águas, 1 em Ciências Agrárias, 1 em Ciências Biológicas, 1 em Ciências da Terra, 1 em Etnodesenvolvimento, 1 em Gestão Ambiental, 1 em Saúde e 1 em Tecnologia da Informação; a Fundação Universidade Federal do Pampa (UNIPAMPA) com 1 curso em Ciência e Tecnologia; a Universidade Federal de Alfenas (UNIFAL-MG) com 2 cursos, 1 em Ciência e Economia e 1 em Ciência e Tecnologia; a Universidade Federal Rural do Semi-Árido (UFERSA) com 7 cursos de Ciência e Tecnologia; a Universidade Federal dos Vales do Jequitinhonha e Mucuri (UFVJM) com 4 cursos, 3 em Ciência e Tecnologia e 1 em Ciências Agrárias; a Universidade Federal do Recôncavo da Bahia (UFRB) com 3 cursos diferentes, 1 em Cultura, Linguagens e Tecnologias Aplicadas, 1 em Energia e Sustentabilidade e, 1 em Saúde, por último, a Universidade Federal do Maranhão (UFMA) com 4 cursos em Ciência e Tecnologia.

Embora, não fazendo parte diretamente do corpo gerador do documento basilar, existem instituições que também aderiram a nova arquitetura acadêmica interdisciplinar, tais como: a Universidade Federal do Pará (UFPA) e a Universidade Federal do Rio Grande do Sul (UFRGS), ambas com 1 curso em Ciência e Tecnologia; a Universidade Federal de São Paulo (UNIFESP) com 2 cursos, 1 em Ciência e Tecnologia e 1 em Ciência e Tecnologia do Mar; a Universidade Federal do Oeste da Bahia (UFOB) com 2 cursos, 1 em Ciência e Tecnologia e 1 em Humanidades.

A posição de liderança pertence a Universidade Federal do Sul da Bahia (UFSB), que ofereceu 12 cursos subdivididos em 4 áreas básicas de ingresso: Humanidades, Artes, Saúde, Ciência, com 3 cursos em cada área. Essa universidade, que iniciou seus cursos no ano de 2014, tem o seu eixo político-pedagógico fundado dentro de uma arquitetura curricular organizada em ciclos de formação, tal qual a definição dos cursos de BI, descrita nos referenciais orientadores. Os referenciais orientadores (BRASIL, 2010) são um documento basilar que contextualizam e fundamentam os cursos de BI e similares, estabelecendo a sua definição, princípios, o perfil dos egressos, escopo, implantação e acompanhamento dos cursos.

Além da quantidade e denominação de cursos de BI, a plataforma eletrônica e-MEC disponibiliza também informações sobre a atos regulatórios como: autorização ou criação do curso, reconhecimento e renovação, alteração de vaga, mudança de endereço, alteração da denominação, retificação, entre outros documentos que são cadastrados na plataforma pela coordenação do curso. Em 2017, do total de 66 cursos de BI, obtiveram-se 44 cursos com documentos de autorização, 22 com documento de criação de curso e 36 com documento de reconhecimento do curso.

As universidades e centros universitários, sejam públicas ou privadas, possuem autonomia para ofertar cursos superiores, independente da autorização do MEC. Mesmo assim, as instituições têm a obrigação de comunicar à Secretaria competente os novos cursos ofertados. A comunicação deve ser feita no prazo de 60 dias, para que sejam realizadas a supervisão, avaliação e, posterior, reconhecimento dos cursos atendendo o artigo 40 do Decreto n. 9.235/2017 (BRASIL, 2017b). Esse período refere-se ao prazo contado a partir da data de criação do curso. No tocante o reconhecimento do curso, a IES deve protocolar o pedido no período e na forma estabelecido pelo MEC, condição necessária para a validação nacional de seus diplomas.

No que tange à espacialização regional versus número de vagas autorizadas, tem-se na figura 1 , 12 cursos em 4 capitais, e na figura 2, os demais 54 cursos disseminados no interior de 8 estados. Neste panorama, são 19 IES que apresentam 66 cursos de BI, autorizando 13.760 vagas. 
Figura 1 - Oferta dos cursos bacharelados interdisciplinares nas capitais, segundo quantidade de vagas e cursos

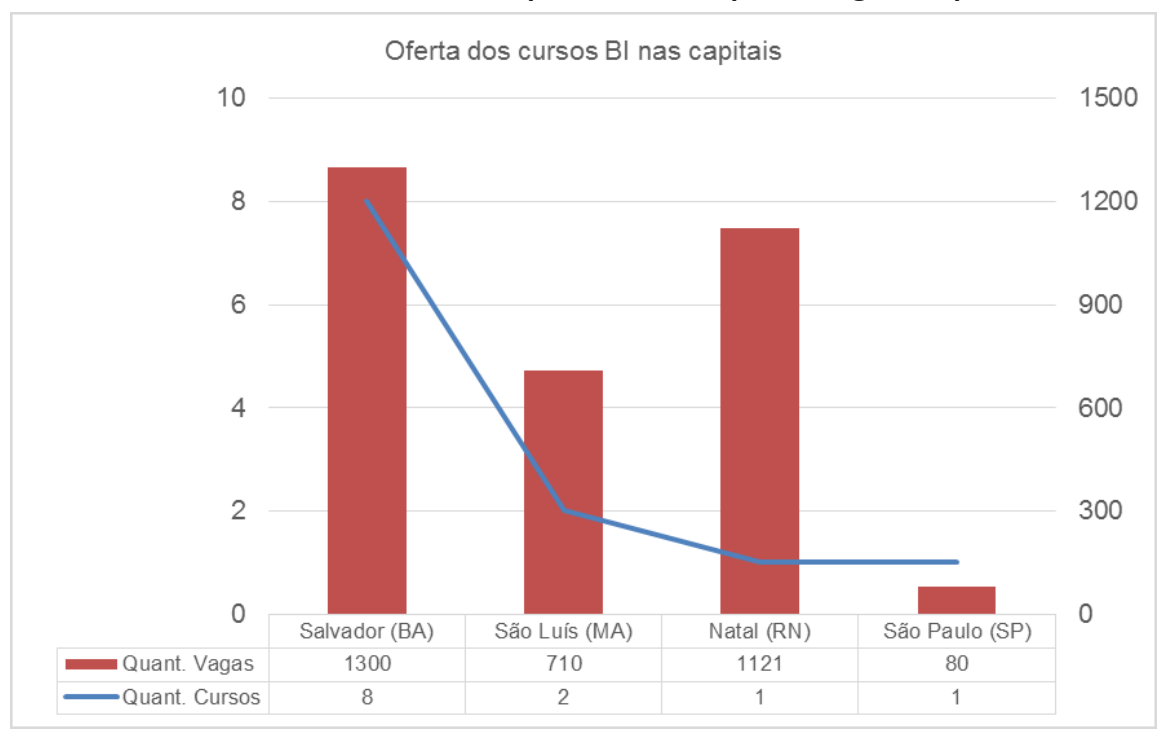

Fonte: Elaborada pelos autores, consulta plataforma eletrônica e-MEC (BRASIL, 2017a)

Figura 2 - Interiorização dos cursos bacharelados interdisciplinares nos estados, segundo quantidade de vagas e cursos

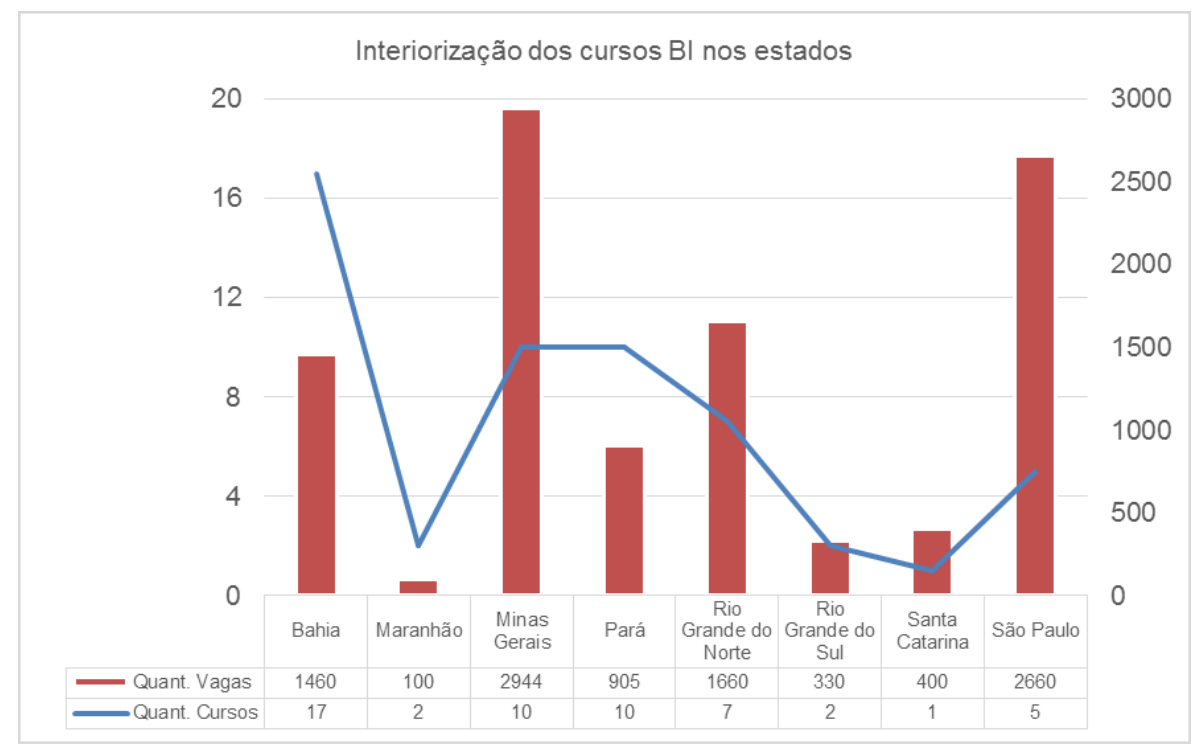

Fonte: Elaborada pelos autores, consulta plataforma eletrônica e-MEC (BRASIL, 2017a)

No cadastro e-MEC, o estado que inspira a oferta em quantidade de cursos de Bl é a Bahia, onde foram implantados 25 cursos, com 2.760 vagas, nas grandes áreas de conhecimento, como: humanidades, artes, saúde, ciência e tecnologia. Deste total, 17 cursos estão situados fora da capital, com implantação de novas IFES, e há 8 cursos na UFBA, em Salvador, o que representa 1.300 vagas. Segundo Almeida Filho (2007), esta nova modalidade de curso superior, os bacharelados interdisciplinares, vem para fomentar a formação dos estudantes universitários em eixos ou temas relevantes da cultura contemporânea, permitindo reestruturação da arquitetura curricular ao torná-la flexível.

No movimento de interiorização da oferta de vagas públicas, 2 estados disponibilizam 3.849 vagas nos cursos de BI, são eles: Minas Gerais, com 2.944 vagas, e Pará, com 905 vagas; sendo que cada um destes estados conta com 10 cursos nesse novo regime de formação estruturada em ciclos introduzidos pelo Processo de Bolonha. Enquanto isso, o Rio Grande do Norte tem 9 cursos: um 
curso na UFRN, na capital Natal, com 1.121 vagas, e os demais na UFERSA, os quais somam 1.660 vagas. Os outros - cerca de $20 \%$ dos cursos de graduação interdisciplinar no Brasil - estão difundidos em 4.280 vagas autorizadas entre: o estado de São Paulo, com 2.740 vagas entre 6 cursos, sendo 1 na capital, com 80 vagas; o Maranhão, com 4 cursos, os quais totalizam 810 vagas, sendo 2 cursos na capital São Luís, somando 710 vagas; e a Região Sul, com um total de 730 vagas distribuídas entre 2 estados, o Rio Grande do Sul que apresenta 2 cursos e soma um total de 330 vagas, e Santa Catarina com 1 curso de 400 vagas, ambos no interior dos respectivos estados.

$\mathrm{Na}$ análise dos dados do e-MEC percebe-se que não existe um número médio único de vagas autorizadas por curso. Isso significa que cada instituição pública apresenta autonomia para criar e estabelecer o número de vagas. Outra constatação realizada a partir da análise é de que a região centro-oeste não apresenta cursos de BI.

Conforme Veras, Lemos e Macedo (2015) os cursos de Bl correspondem a um novo modelo educacional composto por três ciclos que inserem metodologias cujo foco é a interdisciplinaridade e a autonomia do alunado no processo de construção do conhecimento; além de estarem vinculadas à expansão do número de vagas e à inclusão de novas classes sociais no ensino superior.

Cabe salientar que os dados quantitativos de frequência aqui apresentados referem-se ao primeiro ciclo dos bacharelados interdisciplinares. Quando o estudante universitário conclui o curso de $\mathrm{BI}$, é opcional direcionar-se ao segundo ciclo de sua formação em área específica existente no campus, por meio de um processo seletivo interno da IES. O terceiro ciclo foge ao escopo deste artigo, pois se refere aos cursos de pós-graduação stricto senso.

As IES, após a avaliação realizada in loco podem receber um Conceito de Curso (CC) que varia de 1 a 5, de acordo com as condições relacionadas ao perfil dos docentes, à organização didático-pedagógica e à sua infraestrutura. Esse é um indicador de qualidade do MEC para os cursos de graduação, incluindo os Bacharelados Interdisciplinares.

De acordo com cadastro e-MEC, tem-se 45 cursos de BI distribuídos nas 18 IES, os quais apresentaram conceito de curso (CC) que varia de 2 a 5, entre os anos de 2010 e 2017. Outros 21 cursos ainda não foram avaliados, sendo que destes, 6 cursos sequer iniciaram, segundo a plataforma eletrônica.

O primeiro curso de BI avaliado foi em Ciência e Tecnologia, da UFABC, em 2010, o qual recebeu conceito 5. No ano de 2011, o segundo curso de BI avaliado foi o de Artes e Design, da UFJF, o qual recebeu o conceito 4. Em 2012, houve o terceiro curso avaliado, em Artes, da UFBA, o qual obteve conceito 4.

No ano de 2013 ocorreu uma ampliação da avaliação dos cursos de BI, onde 6 cursos receberam conceito 4: 2 cursos em Humanidades, da UFBA; 1 curso em Saúde, da UFBA; 1 curso de Ciências Humanas, da UFJF; e outros 2 cursos em Ciência e Tecnologia, um deles oferecido pela UFVJM e o outro pela UFERSA. No mesmo ano, 2 cursos em Ciência e Tecnologia, um da UFBA e o outro da UFVJM, obtiveram conceito 3.

A quantidade mais significativa em avaliações foi no ano de 2014, totalizando 18 cursos distribuídos entre $10 \mathrm{IES}$, em 6 estados, os quais estão relacionadas na tabela 1, com Conceitos de Curso (CC) variando de 2 a 5. Em resumo, ressalta-se a presença de 3 cursos com nota máxima, igual a 5, 9 cursos com CC igual a 4, e 5 cursos com CC igual a 3, que é considerado satisfatório. A UFOPA teve baixo desempenho no curso de BI em Ciências da Terra, avaliado com CC igual a 2. 
Tabela 1 - Cursos de BI das IES com respectivos Conceitos de Cursos (CC) em 2014

\begin{tabular}{l|c}
\hline Nome do Curso (IES) & CC \\
\hline Ciências do Trabalho (DIEESE) & 5 \\
\hline Ciência e Tecnologia (UFABC) & 5 \\
\hline Ciências e Humanidades (UFABC) & 3 \\
\hline Artes (UFBA) & 3 \\
\hline Ciência e Tecnologia (UFBA) & 4 \\
\hline Saúde (UFBA) & 4 \\
\hline Ciência e Economia (UNIFAL-MG) & 4 \\
\hline Ciência e Tecnologia (UNIFAL-MG) & 4 \\
\hline Mobilidade (UFSC) & 3 \\
\hline Ciência e Tecnologia (UFSJ) & 4 \\
\hline Ciência e Tecnologia (UNIFESP) & 2 \\
\hline Ciências da Terra (UFOPA) & 3 \\
\hline Ciência e Tecnologia (UFOPA) & 3 \\
\hline Ciência e Tecnologia das Águas (UFOPA) & 3 \\
\hline Ciência e Tecnologia (UFRN) & 4 \\
\hline Ciência e Tecnologia (UFERSA) - campus Angicos - RN & 4 \\
\hline Ciência e Tecnologia (UFERSA) - campus Caraúbas - RN & 4 \\
\hline Ciência e Tecnologia (UFERSA) - campus Angicos - RN & 5 \\
\hline
\end{tabular}

Fonte: Elaborada pelos autores, consulta plataforma eletrônica e-MEC (BRASIL, 2017a)

No ano de 2015, na plataforma eletrônica e-MEC existem 5 cursos cadastrados que foram avaliados, tais como: Ciências Agrárias da UFOPA com CC igual 3, com CC igual a 4 os cursos de Biossistemas da UFSJ, Ciência e Tecnologia da UFERSA, Ciência e Tecnologia da UNIPAMPA e com CC igual a 5, Ciência e Tecnologia do Mar da UNIFESP.

Em 2016, apenas um curso de BI em Ciência e Tecnologia, da UFERSA, foi avaliado com CC igual a 4. Situação diferente ocorreu no ano de 2017, conforme a tabela 2, quando foi observado um incremento de 10 cursos de $\mathrm{BI}$ avaliados, conforme o cadastro e-MEC, os quais estão distribuídos entre 6 universidades federais e cujos CC variam entre 3 e 4.

Tabela 2 - Cursos de BI das IFES com respectivos Conceitos de Cursos (CC) em 2017

\begin{tabular}{l|c}
\hline Nome do Curso (IES) & CC \\
\hline Ciência e Tecnologia (UFPA) & 3 \\
\hline Ciência e Tecnologia (UFMA) - campus São Luís - MA & 3 \\
\hline Ciência e Tecnologia (UFMA) - campus Balsas - MA & 3 \\
\hline Ciência e Tecnologia (UFVJM) & 3 \\
\hline Ciências (UFSB) & 3 \\
\hline Ciências Agrárias (UFVJM) & 3 \\
\hline Ciência e Tecnologia (UFRGS) & 4 \\
\hline Cultura, Linguagens e Tecnologias Aplicadas (UFRB) & 4 \\
\hline Energia e Sustentabilidade (UFRB) & 4 \\
\hline Humanidades (UFSB) & 4 \\
\hline
\end{tabular}

Fonte: Elaborada pelos autores, consulta plataforma eletrônica e-MEC (BRASIL, 2017a) 
As categorias analisadas, como número de instituições de ensino superior, os respectivos atos regulatórios, a espacialização regional versus o número de vagas autorizadas e o conceito de curso até o ano de 2017, permitiram uma visualização desta modalidade de graduação plena - os cursos bacharelados interdisciplinares - oferecida por universidades no Brasil. Além de um panorama acerca da articulação dos saberes e conhecimentos, projetando um horizonte inovador impetrado nesta nova arquitetura acadêmica interdisciplinar, após 10 anos da implantação do REUNI, em 2007.

\section{Considerações finais}

Os cursos bacharelados interdisciplinares no Brasil foram uma experiência oriunda de alguns pesquisadores, educadores e gestores, em meados dos anos 1990. A iniciativa deu-se através de alguns atores que, posteriormente, constituíram um grupo de trabalho homologado pelo o Ministério da Educação, cujo objetivo foi elaborar diretrizes pedagógicas e metodológicas para implantação e implementação desses cursos nas universidades brasileiras.

Com a ampliação da oferta de educação superior no Brasil e a expansão das universidades federais, duas universidades públicas foram implantadas com estas características inovadoras na formação do alunado, a UFABC, em São Paulo, e a UFSB, no Sul da Bahia. Em 2017, após 10 anos de implantação do REUNI, constatou-se que houve avanços, porém incipientes ainda, pois os desafios continuam sendo inúmeros: docentes pouco capacitados para lidar com uma formação pedagógica diferente, estudantes resistentes à proposta e pouco preparados no ensino médio para lidar com as metodologias inovadoras no ensino superior dos cursos de bacharelados interdisciplinares.

O modelo pedagógico das universidades brasileiras é impetrado numa lógica centrada no docente, corroborando para uma formação especializada, técnica e pouca generalista. Portanto, indo na contramão da proposta pedagógica dos cursos de bacharelados interdisciplinares.

Outro aspecto, averiguado no estudo foi que os cursos de Bl estão centrados na região sudeste, acompanhada da região sul e nordeste. Há carência de implantação dos cursos de BI nas regiões centro-oeste e norte no Brasil. Embora a UFOPA, que está situado na região norte, tenha-se destacado com a implantação de 9 cursos na região oeste do Pará.

Houve universidades brasileiras, como: UFRJ, UNILAB e UFFS, as quais participaram do grupo de trabalho do documento basilar para a elaboração dos Referenciais Orientadores, mas que não aderiram à propsota de cursos de $\mathrm{BI}$, ao menos durante a realização desta pesquisa. Esses centros de excelência têm se manifestado de forma tímida em relação a inovação do modelo universitário brasileiro. A baixa adesão dessas IES enfraquece a proposta dos cursos de BI no Brasil. Ademais, a baixa adesão das IES privadas é outro ponto culminante para a fragilização desse modelo, pois no panorama há apenas um curso na DIEESE.

As avaliações ou atos regulatórios realizados pelo Instituto Nacional de Estudos e Pesquisas Educacionais Anísio Teixeira (INEP), no que tange os cursos de Bl existentes no Brasil, sinalizam que há qualidade nesses cursos, numa margem conceitual de suficiente a muito bom (conceitos de curso de 2 a 5). Essa constatação serve de estímulo para as demais IES implantarem os cursos de $\mathrm{BI}$, bem como incentivo ao estado fomentar os recursos físicos, operacionais e humanos necessários à instalação de novos cursos de $\mathrm{BI}$ no Brasil.

Entretanto, há também pouco investimento do MEC, no que diz respeito às políticas públicas educacionais de incremento e investimento nos bacharelados interdisciplinares no Brasil. Há certo descaso nas políticas para incentivo à implantação e implementação de novos cursos de Bl, bem como não há uma sinalização ou uma avaliação mais contundente do MEC, em termos de avanços e fragilidades, que esclareça de fato para a sociedade brasileira o que se potencializou nesta proposta. 
No que diz respeito aos indicadores, o que se tem de avaliação é ínfimo, concentrando basicamente em estudos centrados em pesquisadores que se interessam pela temática. Urge um esclarecimento de fato e um maior incentivo do governo federal para que se haja uma maior adesão das IES na implantação e implementação dos cursos de BI no Brasil.

\section{Referências}

ALMEIDA FILHO, N. As três culturas na universidade nova. Ponto de Acesso, Salvador, v.1, n.1, p. 5-15, jun. 2007.

ALMEIDA FILHO, N. Interdisciplinaridade na Universidade Nova: Desafios para a Docência. In: CERVI, G.; RAUSCH, R. B. Docência Universitária: Concepções, Experiências e Dinâmicas de Investigação. Blumenau: Meta Editora, 2014, p. 21-28.

ALVARENGA, A. T. et al. Histórico, fundamentos filosóficos e teórico-metodológicos da interdisciplinaridade. In: PHILIPPI JR., A.; NETO, A. J. S. Interdisciplinaridade em Ciência, Tecnologia e Inovação. Barueri, SP: Manole, 2011, p. 3-68.

BRASIL. Portaria Normativa $\mathbf{n}^{\circ} \mathbf{4 0}$, de 12 de dezembro de 2007. Institui o e-MEC, sistema eletrônico de fluxo de trabalho e gerenciamento de informações relativas aos processos de regulação da educação superior no sistema federal de educação. Brasília: DOU Diário Oficial da União. Publicado no DOU n. ${ }^{\circ} 239$, de 13.12.2007, seção 1, p. 39-43. Disponível em: http://download.inep.gov.br/educacao_superior/censo_superior/legislacao/2007/ portaria_40_12122007.pdf. Acesso em: 20/10/2017.

BRASIL. Referenciais orientadores para os bacharelados interdisciplinares e similares. Documento elaborado pelo Grupo de Trabalho instituído pela Portaria SESu/MEC n 383, de 12.04.2010. Brasília: Ministério da Educação. Secretaria de Educação Superior, 2010. Disponível em: http://reuni.mec.gov.br/images/stories/pdf/novo\%20-\%20bacharelados\%20interdisciplinares\%20-\%20referenciais\%20orientadores\%20\%20novembro_2010\%20 brasilia.pdf. Acesso em: 20/10/2017.

BRASIL. Plataforma e-MEC. Cadastro Nacional de Cursos e Instituições de Educação Superior, Cadastro e-MEC. Brasília: Ministério da Educação, 2017a. Disponível em: https://emec.mec.gov.br/ Acesso em: 20/10/2017.

BRASIL. Decreto 9.235 de 15 de dezembro de 2017. Dispõe sobre o exercício das funções de regulação, supervisão e avaliação das instituições de educação superior e dos cursos superiores de graduação e de pós-graduação no sistema federal de ensino. Brasília: Presidência da República, 15 de dezembro de 2017b; $196^{\circ}$ da Independência e $129^{\circ}$ da República. Disponível em: http://www.planalto.gov.br/ccivil_03/_ato2015-2018/2017/decreto/D9235. htm. Acesso em: 20/10/2017.

FAVERO, M. A Universidade no Brasil: das Origens à Reforma Universitária de 1968. Educar, Curitiba, Editora UFPR, n. 28, p. 17-36, 2006.

HECKHAUSEN, H. Discipline et interdisciplinarité. In: CERI, L'Interdisciplinarité. Problèmes d'Enseignement et de Recherche dans les Universités. Paris: OCDE, p. 83-90, 1972.

KUHN, T. As estruturas das revoluções científicas. São Paulo: Perspectiva, 2003.

MOREIRA, H.; CALEFFE, L. G. Metodologia da Pesquisa para o Professor Pesquisador. 2. ed. Rio de Janeiro: Lamparina Editora, 2006.

PAIM, A. A UDF e a idéia de universidade. Rio de Janeiro: Tempo Brasileiro, 1981. 
SANTOS, B. D. S. Um Discurso sobre as Ciências. 5. ed. São Paulo: Cortez, 2008.

UNESCO. Declaración mundial sobre la educación superior en el siglo XXI: visión y acción. Paris: UNESCO, 1998.

VERAS, R. M.; LEMOS, D. V. da S.; MACEDO, B. T. F. A trajetória da criação dos Bacharelados Interdisciplinares na Universidade Federal da Bahia. Avaliação, Campinas; Sorocaba, SP, v. 20, n. 3, p. 621-641, nov. 2015. 